\title{
Classical and Quantum Mechanics of a Charged Particle in Oscillating Electric and Magnetic Fields
}

\author{
V.L.B. de Jesus, A.P. Guimarães, and I.S. Oliveira \\ Centro Brasileiro de Pesquisas Físicas \\ Rua Dr. Xavier Sigaud 150, Rio de Janeiro - 22290-180, Brazil
}

Received 27 May, 1999

\begin{abstract}
The motion of a particle with charge $q$ and mass $m$ in a magnetic field given by $\mathbf{B}=\mathbf{k} B_{0}+$ $B_{1}[\mathbf{i} \cos (\omega t)+\mathbf{j} \sin (\omega t)]$ and an electric field which obeys $\nabla \times \mathbf{E}=-\partial \mathbf{B} / \partial t$ is analyzed classically and quantum-mechanically. The use of a rotating coordinate system allows the analytical derivation of the particle classical trajectory and its laboratory wavefunction. The motion exhibits two resonances, one at $\omega=\omega_{c}=-q B_{0} / m$, the cyclotron frequency, and the other at $\omega=\omega_{L}=-q B_{0} / 2 m$, the Larmor frequency. For $\omega$ at the first resonance frequency, the particle acquires a simple closed trajectory, and the effective hamiltonian can be interpreted as that of a particle in a static magnetic field. In the second case a term corresponding to an effective static electric field remains, and the particle orbit is an open line. The particle wave function and eigenenergies are calculated.
\end{abstract}

PACS: 41.75.A, 52.50.G, 03.50.D, 12.20

Keywords: magnetic resonance, ion trapping, isotope separation, magnetic confinement

\section{Introduction}

The dynamics of charged particles in electric and magnetic fields is of both academic and practical interest. The areas where this problem finds applications include the development of cyclotron accelerators [1], free electron lasers [2], plasma physics [4] and so on.

In this paper one considers the classical and quantum dynamics of a particle with charge $q$ and mass $m$ acted by a magnetic field given by

$$
\mathbf{B}=\mathbf{k} B_{0}+B_{1}[\mathbf{i} \cos (\omega t)+\mathbf{j} \sin (\omega t)]
$$

and an electric field which relates to $\mathbf{B}$ through the Faraday law:

$$
\nabla \times \mathbf{E}=-\frac{\partial \mathbf{B}}{\partial t}
$$

Both fields can be derived from the vector potential:

$$
\mathbf{A}=-\frac{1}{2} \mathbf{R} \times \mathbf{B}
$$

When $B_{1}$ is given by two pairs of crossed Helmholtz coils, the approximation of homogeneity implicit in equation (1) is valid in a region of about $20 \%$ of the volume enclosed by the coils. Similar assumption have been taken by other authors $[2,3]$.

The classical equations of motion can be obtained from the lagrangian:

$$
\begin{gathered}
\mathcal{L}=\frac{m}{2}\left(\dot{X}^{2}+\dot{Y}^{2}+\dot{Z}^{2}\right)+\frac{q}{2} \dot{X}\left[Z B_{1} \sin (\omega t)-Y B_{o}\right]+ \\
+\frac{q}{2} \dot{Y}\left[X B_{o}-Z B_{1} \cos (\omega t)\right]+\frac{q}{2} \dot{Z}\left[Y B_{1} \cos (\omega t)-X B_{1} \sin (\omega t)\right]
\end{gathered}
$$

whereas to study the quantum dynamics we need the hamiltonian:

$$
\begin{gathered}
\mathcal{H}=\frac{1}{2 m}\left(P_{x}^{2}+P_{y}^{2}+P_{z}^{2}\right)-\frac{q}{2 m}\left[B_{1} \cos (\omega t) L_{x}+B_{1} \sin (\omega t) L_{y}+B_{0} L_{z}\right]+ \\
+\frac{q^{2}}{8 m}\left\{B_{0}^{2}\left(X^{2}+Y^{2}\right)+B_{1}^{2} Z^{2}-2 B_{0} B_{1} Z[X \cos (\omega t)+Y \sin (\omega t)]+\right. \\
\left.+B_{1}^{2}[Y \cos (\omega t)-X \sin (\omega t)]^{2}\right\}
\end{gathered}
$$




\section{The classical motion}

The classical equations of motion can be easily obtained from (4). In order to eliminate the time dependence of the lagrangian, we perform the following transformation of coordinates:

$$
\begin{gathered}
\mathbf{i}=\mathbf{i}^{\prime} \cos (\omega t)-\mathbf{j}^{\prime} \sin (\omega t) \\
\mathbf{j}=\mathbf{i}^{\prime} \sin (\omega t)+\mathbf{j}^{\prime} \cos (\omega t) \\
\mathbf{k}=\mathbf{k}^{\prime}
\end{gathered}
$$

In the nuclear magnetic resonance (NMR) literature [5] these transformations are interpreted as leading to a system of reference which rotates with angular frequency $\omega$ in respect to the laboratory coordinate sys- tem.

From (6), using lower case to indicate the variables in the rotating system, the effective lagrangian becomes:

$$
\begin{gathered}
\mathcal{L}_{\text {eff }}=\frac{m}{2}\left(\dot{x}^{2}+\dot{y}^{2}+\dot{z}^{2}\right)-\frac{q}{2} \dot{x} y\left(B_{\circ}+\frac{2 \omega}{\gamma}\right)+\frac{q}{2} \dot{y}\left[x\left(B_{o}+\frac{2 \omega}{\gamma}\right)-z B_{1}\right]+ \\
+\frac{q \dot{z} y B_{1}}{2}+\frac{q \omega}{2}\left(B_{o}+\frac{\omega}{\gamma}\right)\left(x^{2}+y^{2}\right)-\frac{q \omega B_{1} x z}{2}
\end{gathered}
$$

This lagrangian can be written in the usual compact form:

$$
\mathcal{L}_{e f f}=T+q \dot{\mathbf{r}} \cdot \mathbf{A}_{e f f}-q \phi_{e f f}
$$

where $T$ is the particle kinetic energy, $\mathbf{A}_{\text {eff }}$ the effective vector potential given by:

$$
\mathbf{A}_{e f f}(\mathbf{r})=-\frac{1}{2} \mathbf{r} \times \mathbf{B}_{e f f},
$$

with $\mathbf{B}_{\text {eff }}$ being the effective magnetic field (defined below), and the effective scalar potential:

$$
\phi_{e f f}(\mathbf{r})=\frac{1}{2} \omega B_{1} x z-\frac{\omega}{2}\left(B_{o}+\frac{\omega}{\gamma}\right)\left(x^{2}+y^{2}\right)
$$

where $\gamma=q / m$ is the particle charge-to-mass ratio. Thus, one has for the particle in the rotating frame the following equations of motion:

$$
\begin{aligned}
& \ddot{x}=\gamma\left[\dot{y}\left(B_{o}+\frac{2 \omega}{\gamma}\right)+x \omega\left(B_{o}+\frac{\omega}{\gamma}\right)-\frac{z \omega B_{1}}{2}\right] \\
& \ddot{y}=\gamma\left[\dot{z} B_{1}-\dot{x}\left(B_{\circ}+\frac{2 \omega}{\gamma}\right)+y \omega\left(B_{\circ}+\frac{\omega}{\gamma}\right)\right]
\end{aligned}
$$

$$
\ddot{z}=-\gamma\left[\dot{y} B_{1}+\frac{x \omega B_{1}}{2}\right]
$$

It is useful to define an effective electric field $\mathbf{E}_{\text {eff }}$. The expressions for the effective fields are:

$$
\begin{gathered}
\mathbf{E}_{e f f}=\left[x \omega\left(B_{o}+\frac{\omega}{\gamma}\right)-\frac{z \omega}{2} B_{1}\right] \mathbf{i}^{\prime}+ \\
+\left[y \omega\left(B_{o}+\frac{\omega}{\gamma}\right)\right] \mathbf{j}^{\prime}-\frac{x \omega B_{1}}{2} \mathbf{k}^{\prime} \\
\mathbf{B}_{e f f}=B_{1} \mathbf{i}^{\prime}+\left(B_{o}+\frac{2 \omega}{\gamma}\right) \mathbf{k}^{\prime}
\end{gathered}
$$

Therefore, for a fixed value of $\omega$, each particle with a given charge-to-mass ratio, $\gamma$, will feel different effective fields. Note that $\mathbf{B}_{\text {eff }}$ differs from that in the NMR case by a factor ' 2 ' in the "apparent field" $\omega / \gamma[5]$. With these definitions, the form of the Lorentz force is preserved in the rotating system:

$$
\mathbf{F}_{e f f}=q \mathbf{E}_{e f f}+q \mathbf{v} \times \mathbf{B}_{e f f}
$$

One can clearly see from (11) that there are two resonance frequencies in the motion: one at $\omega=\omega_{c}=$ $-q B_{0} / m$, the cyclotron frequency, and another at $\omega_{L}=$ $-q B_{0} / 2 m$, the Larmor frequency. For a frequency equal 
to the first one $\left(\omega_{c}\right)$ the particle feels the following effective fields:

$$
\begin{gathered}
\mathbf{E}_{e f f}=-\frac{1}{2} \omega B_{1}\left[z \mathbf{i}^{\prime}+x \mathbf{k}^{\prime}\right] \\
\mathbf{B}_{e f f}=B_{1} \mathbf{i}^{\prime}-B_{o} \mathbf{k}^{\prime}
\end{gathered}
$$

and for the second frequency $\left(\omega=\omega_{L}\right)$,

$$
\begin{gathered}
\mathbf{E}_{e f f}=\frac{1}{2}\left[x \omega B_{o}-z \omega B_{1}\right] \mathbf{i}^{\prime}+\frac{1}{2} y \omega B_{o} \mathbf{j}^{\prime}-\frac{1}{2} x \omega B_{1} \mathbf{k}^{\prime} \\
\mathbf{B}_{\text {eff }}=B_{1} \mathbf{i}^{\prime}
\end{gathered}
$$

Now we consider a particle incident in region of fields $B_{0}$ and $B_{1}$, at the origin of the coordinate system, with initial velocity parallel to $\mathbf{B}_{0}$. That is, $x(0)=y(0)=z(0)=0, v_{x}(0)=v_{y}(0)=0$, and $v_{z}(0)=v_{0}$. As in usual NMR, one makes the approximation $B_{0} \gg B_{1}$. In this limit, it is easy to verify by direct substitution the following solutions of (11), for $\omega=\omega_{c}$ :

$$
\begin{array}{r}
x(t) \approx \frac{\sqrt{3} v_{o}}{3 \omega_{c}} \sin \left(\frac{\sqrt{3} \omega_{1} t}{2}\right)-\frac{\omega_{1} v_{o}}{2 \omega_{c}^{2}} \sin \left(\omega_{c} t\right) \\
y(t) \approx-\frac{2 v_{o}}{3 \omega_{1}}\left[\cos \left(\frac{\sqrt{3} \omega_{1} t}{2}\right)-1\right]-\frac{\omega_{1} v_{o}}{2 \omega_{c}^{2}} \cos \left(\omega_{c} t\right) \\
z(t) \approx \frac{2 \sqrt{3} v_{o}}{3 \omega_{1}} \sin \left(\frac{\sqrt{3} \omega_{1} t}{2}\right)
\end{array}
$$

where $\omega_{1} \equiv \gamma B_{1}$. For $\omega=\omega_{L}$ :

$$
\begin{gathered}
x(t) \approx \frac{\omega_{1} v_{0}}{\omega_{L}^{2}} \sin \left(\omega_{L} t\right)-\frac{v_{0}}{\omega_{L}} \sinh \left(\frac{\omega_{1} t}{2}\right) \\
y(t) \approx \frac{\omega_{1} v_{0}}{2 \omega_{c}^{2}} \cos \left(\omega_{L} t\right)+\frac{v_{0} \omega_{1}}{\omega_{L}^{2}} \cosh \left(\frac{\omega_{1} t}{2}\right) \\
z(t) \approx \frac{2 v_{0}}{\omega_{1}} \sinh \left(\frac{\omega_{1} t}{2}\right)
\end{gathered}
$$

The detailed calculation for the obtention of these solutions is given in ref. [8].

Then, we see that whereas for $\omega=\omega_{c}$ the solutions are purely trigonometric functions, for $\omega=\omega_{L}$ there is a mixture of trigonometric and hyperbolic functions. This means that the trajectory of the particle will be a closed path in the first case, and an open line in the second. For a general value of $\omega$, which can be very close to $\omega_{c}$, there will also be an exponential drift. As an example, Figure 1 shows the trajectories of particles in a beam containing triply ionized isotopes of uranium. The respective charge-to-mass ratios in $\mathrm{MHz} / \mathrm{T}$ are as follow: $\gamma\left({ }^{233} \mathrm{U}\right)=1.242 ; \gamma\left({ }^{234} \mathrm{U}\right)=1.237$; $\gamma\left({ }^{235} \mathrm{U}\right)=1.231 ; \gamma\left({ }^{236} \mathrm{U}\right)=1.226$ and $\gamma\left({ }^{238} \mathrm{U}\right)=1.216$. For this simulation we set $v_{0}=10^{4} \mathrm{~m} / \mathrm{s}, B_{0}=1 \mathrm{~T}$, $B_{1}=0.01 \mathrm{~T}$. The oscillating field frequency is tuned to the cyclotron frequency of the isotope ${ }^{235} \mathrm{U}$, that is, $\omega=-1.231 \mathrm{MHz}$. The drawing is in the rotating system. One can have a picture of the trajectories in the laboratory system by rotating the figure about the $z$ axis. Note that each isotope, according to Eqs. (12) and (13), feels different effective fields. This causes the lighter isotopes to deviate in opposite directions in respect to the heavier ones.

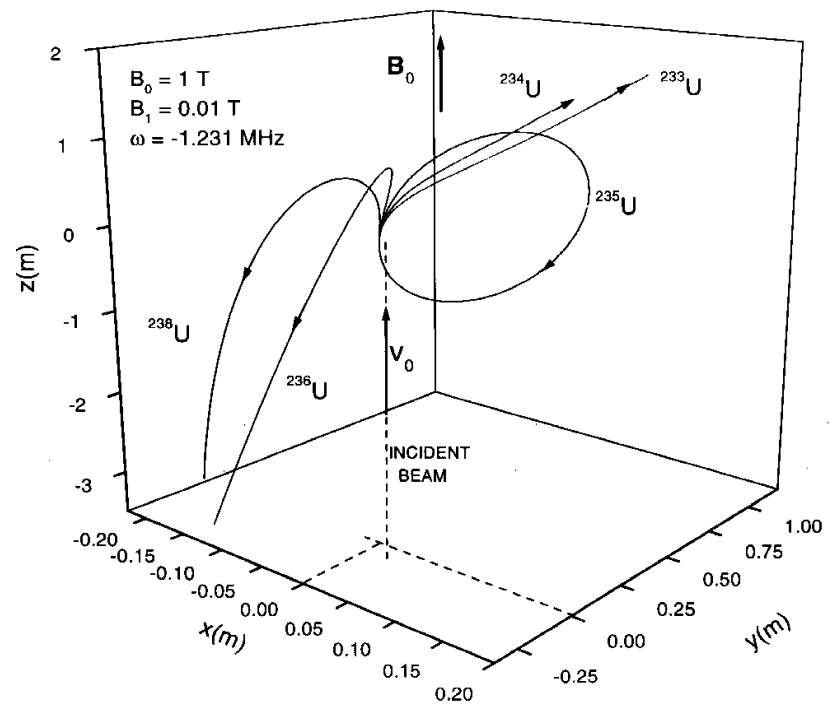

Figure 1. Trajectories of triply ionized Uranium isotopes in oscillating fields "tuned" to the ${ }^{235} \mathrm{U}$ isotope resonance $(-1.231 \mathrm{MHz})$ in the rotating coordinate system. The static field is along the $+z$-axis, and the oscillating magnetic field is on the $x y$-plane. The initial velocity of the particles is $v_{0}=10^{4} \mathrm{~m} / \mathrm{s}$, along the direction of the static field. The orbit of the resonant particle is closed, whereas the nonresonant ones drift away. The trajectories in the laboratory system are obtained rotating the picture about the $z$-axis. These curves were produced for different lengths of time in order to make them all visible in the same scale.

\section{Quantum description}

In this section we approach the problem from the quantum-mechanical point of view. The transformation to the rotating frame in this case, made directly on the Schrödinger equation, allows the derivation of the laboratory wave function and the particle eigenenergies.

Using the following straightforward relations: 


$$
\begin{gathered}
\text { (i) } X \cos (\omega t)+Y \sin (\omega t)=e^{-i \omega t L_{z} / \hbar} X e^{i \omega t L_{z} / \hbar} \\
\text { (ii) }[Y \cos (\omega t)-X \sin (\omega t)]^{2}=e^{-i \omega t L_{z} / \hbar} Y^{2} e^{i \omega t L_{z} / \hbar} \\
\text { (iii) } X^{2}+Y^{2}=e^{-i \omega t L_{z} / \hbar}\left(X^{2}+Y^{2}\right) e^{i \omega t L_{z} / \hbar} \\
\text { (iv) } Z^{2}=e^{-i \omega t L_{z} / \hbar} Z^{2} e^{i \omega t L_{z} / \hbar} \\
\text { (v) } L_{x} \cos (\omega t)+L_{y} \sin (\omega t)=e^{-i \omega t L_{z} / \hbar} L_{x} e^{i \omega t L_{z} / \hbar} \\
L_{z}=e^{-i \omega t L_{z} / \hbar} L_{z} e^{i \omega t L_{z} / \hbar}
\end{gathered}
$$

where, $L_{x}, L_{y}$ and $L_{z}$ are the components of the canonical angular momentum of the particle, the hamiltonian $\mathcal{H}$ in Eq. (5) becomes ${ }^{1}$ :

$$
\begin{aligned}
e^{i \omega t L_{z} / \hbar} \mathcal{H}(t) e^{-i \omega t L_{z} / \hbar} & \equiv \mathcal{H}^{\prime}=\frac{P^{2}}{2 m}+\frac{m \gamma^{2} B_{0}^{2}}{8}\left(X^{2}+Y^{2}\right)+\frac{m \gamma^{2} B_{1}^{2}}{8}\left(Y^{2}+Z^{2}\right) \\
& -\frac{m \gamma^{2} B_{0} B_{1}}{4} X Z-\frac{\gamma B_{0}}{2} L_{z}-\frac{\gamma B_{1}}{2} L_{x}
\end{aligned}
$$

This hamiltonian represents a charged particle moving in a static magnetic field $\mathbf{B}=B_{1} \mathbf{i}+B_{0} \mathbf{k}$. The above operation can be interpreted as the quantum-mechanical transformation of the hamiltonian to the rotating coordinate system.

Defining the wavefunction $\psi^{\prime}$ through the relation:

$$
\psi=e^{-i \omega t L_{z} / \hbar} \psi^{\prime}
$$

and replacing into the Schrödinger equation one obtains:

$$
\left(\mathcal{H}^{\prime}-\omega L_{z}\right) \psi^{\prime}=i \hbar \frac{\partial \psi^{\prime}}{\partial t} \equiv \mathcal{H}_{e f f}^{\prime} \psi^{\prime}
$$

Since $\mathcal{H}_{\text {eff }}^{\prime}$ is time-independent, the solution of (22) will be:

$$
\psi^{\prime}(t)=e^{-i\left(\mathcal{H}^{\prime}-\omega L_{z}\right) t / \hbar} \psi(0)
$$

and consequently the wavefunction in the laboratory system will be

$$
\psi(t)=e^{-i \omega t L_{z} / \hbar} e^{-i\left(\mathcal{H}^{\prime}-\omega L_{z}\right) t / \hbar} \psi(0)
$$

Note that since $\left[L_{z}, \mathcal{H}^{\prime}\right] \neq 0$, the two exponential operators in Eq. (24) cannot be gathered into one.

Now we shall analyze the properties of $\mathcal{H}_{\text {eff }}^{\prime}$ :

$$
\begin{aligned}
\mathcal{H}_{e f f}^{\prime}= & \frac{P^{2}}{2 m}+\frac{m \gamma^{2} B_{0}^{2}}{8}\left(X^{2}+Y^{2}\right)+\frac{m \gamma^{2} B_{1}^{2}}{8}\left(Y^{2}+Z^{2}\right) \\
& -\frac{m \gamma^{2} B_{0} B_{1}}{4} X Z-\frac{\gamma \Delta B}{2} L_{z}-\frac{\gamma B_{1}}{2} L_{x}
\end{aligned}
$$

where $\Delta B \equiv B_{0}+2 \omega / \gamma$. By adding and subtracting the quantity

$$
\frac{m \gamma^{2}}{8}\left(\frac{4 \omega^{2}}{\gamma^{2}}+\frac{4 \omega B_{0}}{\gamma}\right)\left(X^{2}+Y^{2}\right)-\frac{m \gamma B_{1}}{4} 2 \omega X Z
$$

\footnotetext{
${ }^{1}$ For the quantum treatment we keep capitals throughout the section.
} 
the effective hamiltonian can be re-written as:

$$
\begin{gathered}
\mathcal{H}_{e f f}^{\prime}=\frac{P^{2}}{2 m}+\frac{m \gamma^{2} \Delta B^{2}}{8}\left(X^{2}+Y^{2}\right)+\frac{m \gamma^{2} B_{1}^{2}}{8}\left(Y^{2}+Z^{2}\right) \\
-\frac{m \gamma^{2} \Delta B B_{1}}{4} X Z-\frac{\gamma \Delta B}{2} L_{z}-\frac{\gamma B_{1}}{2} L_{x}+q \frac{\omega}{2}\left[B_{1} X Z-\left(\frac{\omega}{\gamma}+B_{0}\right)\left(X^{2}+Y^{2}\right)\right]
\end{gathered}
$$

which, in turn, has the general form:

$$
\mathcal{H}_{e f f}^{\prime}=\frac{1}{2 m}\left(\mathbf{P}-q \mathbf{A}_{e f f}\right)^{2}+q \phi_{e f f}
$$

where the effective scalar potential is again given by

$$
\phi_{\text {eff }}=\frac{\omega}{2}\left[B_{1} X Z-\left(\frac{\omega}{\gamma}+B_{0}\right)\left(X^{2}+Y^{2}\right)\right]
$$

$\mathbf{A}_{\text {eff }}$ being the effective vector potential. The components of $\mathbf{A}_{\text {eff }}$ can be obtained by commuting $X, Y$ and $Z$ with $\mathcal{H}_{e f f}^{\prime}$, and comparing the result with the definition of the canonical momentum $\mathbf{P}=m \dot{\mathbf{R}}+q \mathbf{A}$. For instance:

$$
\begin{gathered}
i \hbar \dot{X}=\left[X, \mathcal{H}_{e f f}^{\prime}\right]=\frac{1}{2 m} 2 i \hbar P_{x}+\frac{\gamma \Delta B}{2} i \hbar Y \\
P_{x}=m \dot{X}-q \frac{\Delta B}{2} Y
\end{gathered}
$$

Consequently,

$$
A_{e f f, x}=-\frac{\Delta B}{2} Y
$$

Repeating the procedure for the other components, one obtains:

$$
\begin{gathered}
A_{e f f, y}=-\frac{B_{1}}{2} Z+\frac{\Delta B}{2} X \\
A_{e f f, z}=\frac{B_{1}}{2} Y
\end{gathered}
$$

These results are the same as those obtained in ref. [6], the only difference being a factor '2' in the definition of $\Delta B$.

Written in the form of Eq. (26), the hamiltonian exhibits the effects of the electric field. Contrary to what happens when this is neglected [6], it shows two resonance frequencies. At the Larmor frequency, $\Delta B=0$, and the hamiltonian becomes:

$$
\mathcal{H}_{\text {eff }}^{\prime}=\frac{P^{2}}{2 m}+\frac{m \gamma^{2} B_{1}^{2}}{8}\left(Y^{2}+Z^{2}\right)+\frac{\gamma B_{1}}{2} L_{x}-\frac{m \gamma^{2} B_{0} B_{1}}{4} X Z+\frac{m \gamma^{2} B_{0}^{2}}{4}\left(X^{2}+Y^{2}\right)
$$

which represents a particle in a static magnetic field along the $x$ direction, plus an electric field potential. The eigenstates of the particle in this case cannot be easily found. On the other hand, at the cyclotron frequency, the second term of $\phi_{\text {eff }}$ in Eq. (28) vanishes, and the hamiltonian becomes:

$$
\begin{aligned}
\mathcal{H}_{e f f}^{\prime}= & \frac{P^{2}}{2 m}+\frac{m \gamma^{2} B_{0}^{2}}{8}\left(X^{2}+Y^{2}\right)+\frac{m \gamma^{2} B_{1}^{2}}{8}\left(Y^{2}+Z^{2}\right) \\
& -\frac{m \gamma^{2} B_{0} B_{1}}{4} X Z-\frac{\gamma B_{0}}{2} L_{z}+\frac{\gamma B_{1}}{2} L_{x}
\end{aligned}
$$

which represents a particle moving in a static magnetic field $\mathbf{B}_{\text {eff }}=B_{0} \mathbf{k}-B_{1} \mathbf{i}$. This hamiltonian can easily written in a diagonal form by defining the angle

$$
\theta=t g^{-1}\left(\frac{B_{1}}{B_{0}}\right)
$$

between the $z$-axis and $\mathbf{B}_{\text {eff }}$, and writing the operators of $\mathcal{H}_{e f f}^{\prime}$ in (30) in terms of the new coordinates, $X^{\prime}, Y^{\prime}$ and $Z^{\prime}$, where the effective field is axial. 
The particle's eigenenergies are given in this case by:

$$
E_{n}=\frac{P_{z}^{\prime}}{2 m}+\left(n+\frac{1}{2}\right) \hbar \omega_{c}^{\prime}
$$

where $\omega_{c}^{\prime}=\gamma B_{\text {eff }}=\gamma \sqrt{B_{0}^{2}+B_{1}^{2}}$ is the cyclotron frequency about the effective field in the rotating system. From this one sees that the quantization axis can be rotated continuously by changing the angle $\theta$ through the change in the ratio $B_{1} / B_{0}$.

\section{Conclusions}

In this paper we have studied the classical and the quantum dynamics of a charged particle in oscillating magnetic and electric fields which are related through the Faraday law. The equations of motion show two resonance frequencies, one at the Larmor frequency $\left(\omega_{L}\right)$ and another at the cyclotron frequency $\left(\omega_{c}\right)$. When the field frequency equals $\omega_{c}$, the particle is confined to a simple closed trajectory, but when $\omega=\omega_{L}$, it drifts away, the same happening to off resonance particles whose frequencies are very close to $\omega_{c}$. The use of a "rotating coordinate system" such as used in conventional nuclear magnetic resonance allows the derivation of analytical solutions for the equations of motion.

By using the corresponding quantum-mechanical transformation, one finds the exact wavefunction of the particle in the laboratory system. When $\omega=\omega_{c}$, the effective hamiltonian corresponds to that of a charged particle in an static magnetic field. In this case the particle eigenenergies are derived in the rotating coordinate system, and it is shown that the direction of the axis of quantization can be continuously rotated by changing the ratio between the intensities of the fields. On the other hand, when $\omega=\omega_{L}$, the hamiltonian is a mixture of effective magnetic and electric fields, and the eigenenergies cannot be easily derived.

The Hamiltonian (30) predicts the existence of "current echoes", and therefore is in accordance with the results of references [6] and [7]. There is, however, one important difference which appears in the present case where the electric field is considered. Contrary to what happens in [6], the static field term does not vanish at resonance. Thus, in order to describe properly the formation of a current echo in the present situation, one must consider $B_{1} \gg B_{0}$ when the pulse is "on", and obviously $B_{1}=0$ when it is off. Having this in mind, the calculation for the current echo amplitude can be carried out in the same way as described in [6].

\section{Acknowledgements}

The authors are in debt to Prof. W. Baltensperger, Prof. N. V. de Castro Faria and to Dr. S. A. Dias, for their useful suggestions. V. L. B. J. acknowledges the support from CNPq, Brazil.

\section{References}

[1] D.G. Swanson, Rev. Mod. Phys. 67, 837 (1995).

[2] J. Valat, Nuc. Instr. Meth. Phys. Res. A304 502 (1991).

[3] P. Diament, Phys. Rev. A23, 2537 (1981).

[4] K.W. Gentle, Rev. Mod. Phys. 67, 809 (1995).

[5] C.P. Slichter, Principles of Magnetic Resonance (Springer-Verlag, Berlin, 1990) 3rd ed.

[6] I.S. Oliveira, A.P. Guimarães and X.A. da Silva, Phys. Rev. E 55, 2063 (1997).

[7] I.S. Oliveira, Phy. Rev. Lett. 77, 139 (1996).

[8] V.L.B. de Jesus, A.P. Guimarães and I.S. Oliveira, J. Phys. B: At. Mol. Opt. Phys. 31, 2457 (1998). 